\title{
Solution of Moving Boundary Space-Time Fractional Burger's Equation
}

\author{
E. A.-B. Abdel-Salam, ${ }^{1,2}$ E. A. Yousif,, ${ }^{2,3}$ Y. A. S. Arko,, ${ }^{2,4}$ and E. A. E. Gumma ${ }^{2,5}$ \\ ${ }^{1}$ Department of Mathematics, Faculty of Science, Assiut University, New Valley Branch, El-Kharja 72511, Egypt \\ ${ }^{2}$ Department of Mathematics, Faculty of Science, Northern Border University, Arar 91431, Saudi Arabia \\ ${ }^{3}$ Department of Applied Mathematics, Faculty of Mathematical Sciences, University of Khartoum, 11111 Khartoum, Sudan \\ ${ }^{4}$ Department of Mathematics, Faculty of Science, Sudan University of Science and Technology, 11115 Khartoum, Sudan \\ ${ }^{5}$ Department of Mathematics, Faculty of Applied and Pure Science, International University of Africa, 14415 Khartoum, Sudan
}

Correspondence should be addressed to E. A.-B. Abdel-Salam; emad_abdelsalam@yahoo.com

Received 5 March 2014; Accepted 13 August 2014; Published 11 September 2014

Academic Editor: Javier Oliver

Copyright (c) 2014 E. A.-B. Abdel-Salam et al. This is an open access article distributed under the Creative Commons Attribution License, which permits unrestricted use, distribution, and reproduction in any medium, provided the original work is properly cited.

\begin{abstract}
The fractional Riccati expansion method is used to solve fractional differential equations with variable coefficients. To illustrate the effectiveness of the method, the moving boundary space-time fractional Burger's equation is studied. The obtained solutions include generalized trigonometric and hyperbolic function solutions. Among these solutions, some are found for the first time. The linear and periodic moving boundaries for the kink solution of the Burger's equation are presented graphically and discussed.
\end{abstract}

\section{Introduction}

In recent years, fractional differential equations (FDEs) have become one of the most exciting and extremely active areas of research because of their potential applications in physics and engineering. These include electromagnetic, fluid flow, dynamical process in self-similar and porous structures, electrical networks, probability and statistics control theory of dynamical systems, chemical physics, optics, acoustic, viscoelasticity, electrochemistry, and material science [1-9].

There are different kinds of fractional integration and differentiation operators. The most famous one is the RiemannLiouville definition [10-12], which has been used in various fields of science and engineering successfully, but this definition leads to the result that constant function differentiation is not zero. Caputo put definitions which give zero value for fractional differentiation of constant function, but these definitions require that the function should be smooth and differentiable [10-12]. Recently, Jumarie derived definitions for the fractional integral and derivative called modified Riemann-Liouville [13-15], which are suitable for continuous and nondifferentiable functions and give differentiation of a constant function equal to zero. The modified RiemannLiouville fractional definitions are used effectively in many different problems [16-20].

In the literature, there are many effective methods to treat FDEs such as the Adomian decomposition method, the variational iteration method, the homotopy perturbation method, the differential transform method, the finite difference method, the finite element method, the exponential function method [21], the fractional subequation method [22], the $\left(G^{\prime} / G\right)$-expansion method $[23,24]$, and the first integral method [25]. Based on Jumarie's modified Riemann-Liouville derivative and the fractional Riccati equation $D_{x}^{\alpha} F(x)=\sigma+F(x)^{2}$, Zhang and Zhang in [26] introduced the subequation method for solving nonlinear time fractional biological population model and $(4+1)$ dimensional space-time fractional Fokas equation. Guo et al. in [27] improved the subequation method; they obtained the analytical solutions of the space-time fractional WhithamBroer-Kaup and generalized Hirota-Satsuma coupled KdV equations by introducing a new general ansätz. Recently, by extending the fractional Riccati equation in [26] to the more general form $D_{x}^{\alpha} F(x)=A+B F(x)^{2}$, Abdel-Salam and Yousif 
[28] presented the fractional Riccati expansion method to obtain exact solutions of the space-time fractional Kortewegde Vries equation, the spacetime fractional RLW equation, the space-time fractional Boussinesq equation, and the spacetime fractional Klein-Gordon equation. In addition, Li et al. in [29] extended fractional Riccati expansion method for solving the time fractional Burger's equation and the spacetime fractional Cahn-Hilliard equation.

The moving boundary problem is a nonlinear initialboundary value problem that requires extra boundary conditions to determine the motion of the boundary [30, 31]. Moving boundary problems arise in applications that involve time varying geometries. They have numerous applications in areas of physics, engineering, and biology. For example, coating flows, viscous sintering in industrial processes, melting and solidification problems, heat flow and diffusion with phase change, the formation of earth's crust according to plate tectonics theory, and the phenomenon of oxygen diffusion from blood into oxygen consuming tissue give rise to a moving boundary [32-36]. Generally, the solution differs according to the boundary conditions, coordinate dimensions, and the method of solution. Numerous numerical methods have been considered for solving this problem; examples include front-tracking, the front-fixing, the domain-fixing, finite-difference, finite-element, moving grid, phase field, and a fully implicit ghost-cell immersed boundary [37-45]. Atkinson [46] studied time fractional diffusion for the motion of planar boundaries as well as cylindrical and spherical ones, the solution obtained for slow growth by the method of matched asymptotic expansion. Yin and $\mathrm{Xu}$ [47] used two-parameter regular perturbation technique and Fourier-Laplace transform method in finding analytical solution given in terms of the Wright function for a problem of a drug released from a polymeric matrix that can be dissolved into a solvent. Kushwaha and Kumar [48] approximated the solution of a model governed by spacetime fractional derivative for a moving boundary problem which occurs in fluviodeltaic sedimentation process on earth surface. Few researchers investigated some exact analytical solutions using the similarity transformation method and matched asymptotic expansion method. Also, the analytical and numerical solutions of the fractional single phase moving boundary problem were obtained by Li et al. [49].

Burger's equation is a classical nonlinear differential equation which was firstly introduced by Burger in 1948. It is used as a model for many nonlinear physical phenomena such as acoustics, continuous stochastic processes, dispersive water waves, gas dynamics, heat conduction, longitudinal elastic waves in an isotropic solid, number theory, shock waves, and turbulence [50-55]. The space-time fractional Burger's equation, which is a transformed generalization of the Burger's equation, is defined as follows:

$$
D_{t}^{\alpha} u=\sigma D_{x}^{2 \alpha} u+2 \mu u D_{x}^{\alpha} u, \quad 0<\alpha \leq 1,
$$

where $u=u(x, t), \sigma, \mu$ are arbitrary constants and $\alpha$ is the fractional order derivative. The initial-boundary conditions of (1) are

$$
u(x, 0)=h(x), \quad u(s(t), t)=g(t),
$$

where $s(t) \leq x<\infty, t \geq 0, h(x), s(t)$, and $g(t)$ are given functions of their arguments; also, $g(t)$ is continuous and bounded. The function $s(t)$ is assumed to be smooth and it describes the motion of the boundary. It is important to note that when $\alpha=1$ and the motion of the boundary is unknown, one obtains a Stefan problem [56-58]. The aim of the paper is to find the analytic solution of the moving boundary spacetime Burger's equation (1) via variable coefficient fractional Riccati expansion method. To solve (1)-(2), a linear transformation is used to convert a constant coefficient FDE with moving boundary into a variable coefficient FDE with a fixed boundary.

The rest of this paper is organized as follows: the description of the fractional Riccati expansion method with variable coefficients is presented in Section 2. In Section 3, the solution of the moving boundary space-time Burger's equation is studied. In Section 4, discussion and conclusion are presented.

\section{Description of the Fractional Riccati Expansion Method with Variable Coefficients}

In this section, we present the fractional Riccati expansion method with variable coefficients to find exact analytical solutions of nonlinear FDEs. The fractional derivatives are described in sense of the modified Riemann-Liouville derivative defined by Jumarie [59-61] as

$$
\begin{aligned}
& D_{x}^{\alpha} f(x) \\
& = \begin{cases}\frac{1}{\Gamma(1-\alpha)} \int_{0}^{x}(x-\xi)^{-\alpha-1}[f(\xi)-f(0)] d \xi, \\
\frac{1}{\Gamma(1-\alpha)} \frac{d}{d x} \int_{0}^{x}(x-\xi)^{-\alpha}[f(\xi)-f(0)] d \xi, \\
{\left[f^{(\alpha-n)}(x)\right]^{(n)},} & 0<\alpha<1 \\
& n \leq \alpha<n+1, n \geq 1,\end{cases}
\end{aligned}
$$

which has merits over the original one; for example, the $\alpha$ order derivative of a constant is zero. Some properties of the Jumarie's modified Riemann-Liouville derivative are

$$
\begin{aligned}
D_{x}^{\alpha} x^{\gamma} & =\frac{\Gamma(\gamma+1)}{\Gamma(\gamma+1-\alpha)} x^{\gamma-\alpha}, \quad \gamma>0, \\
D_{x}^{\alpha}[f(x) g(x)] & =g(x) D_{x}^{\alpha} f(x)+f(x) D_{x}^{\alpha} g(x), \\
D_{x}^{\alpha} f[g(x)] & =f_{g}^{\prime}[g(x)] D_{x}^{\alpha} g(x)=D_{g}^{\alpha} f[g(x)]\left(g_{x}^{\prime}\right)^{\alpha} .
\end{aligned}
$$

The above properties play an important role in the fractional Riccati expansion method with variable coefficients.

Consider a nonlinear FDE in two variables $x$ and $t$ to be

$$
P\left(u, D_{t}^{\alpha} u, D_{x}^{\alpha} u, D_{t}^{2 \alpha} u, D_{x}^{2 \alpha} u, \ldots\right)=0, \quad 0<\alpha \leq 1,
$$

where $u=u(x, t)$ is an unknown function, $D_{t}^{\alpha} u$ and $D_{x}^{\alpha} u$ are Jumarie's modified Riemann-Liouville derivatives of $u$, 
and $P$ is a polynomial in $u$ and its various partial derivatives, in which the highest order derivatives and nonlinear terms are involved.

Suppose that $u(\xi)$ can be expressed by a finite power series of $F(\xi)$ as

$$
u(\xi)=a_{0}(t)+\sum_{i=1}^{n} a_{i}(t) F^{i}(\xi), \quad a_{n}(t) \neq 0,
$$

where $\xi=k x+f(t), k$ is arbitrary constant and $f(t), a_{i}(t)(i=0,1,2, \ldots, n)$ are arbitrary functions of $t$ to be determined later, $n$ is a positive integer determined by balancing the linear term of the highest order with the nonlinear term in (5), and $F=F(\xi)$ satisfies the following fractional Riccati equation:

$$
D_{\xi}^{\alpha} F=A+B F^{2}, \quad 0<\alpha \leq 1,
$$

where $A$ and $B$ are constants. Using the Mittag-Leffler function in one parameter $E_{\alpha}(x)=\sum_{k=0}^{\infty}\left(x^{k} / \Gamma(1+k \alpha)\right)(\alpha>$ $0)$, we obtain the following solutions of $(7)$.

Case 1. If $A=1$ and $B=1$, then $F=\tan (\xi, \alpha)$.

Case 2. If $A=-1$ and $B=-1$, then $F=\cot (\xi, \alpha)$.

Case 3. If $A=1$ and $B=-1$, then $F=\tanh (\xi, \alpha), F=$ $\operatorname{coth}(\xi, \alpha)$.

Case 4. If $A=1 / 2$ and $B=-1 / 2$, then

$$
F=\frac{\tanh (\xi, \alpha)}{1 \pm \operatorname{sech}(\xi, \alpha)}, \quad F=\operatorname{coth}(\xi, \alpha) \pm \operatorname{csch}(\xi, \alpha) \text {. }
$$

Case 5. If $A=1 / 2$ and $B=1 / 2$, then

$$
\begin{gathered}
F=\frac{\tan (\xi, \alpha)}{1 \pm \sec (\xi, \alpha)}, \quad F=\csc (\xi, \alpha)-\cot (\xi, \alpha), \\
F=\tan (\xi, \alpha) \pm \sec (\xi, \alpha) .
\end{gathered}
$$

Case 6. If $A=-1 / 2$ and $B=-1 / 2$, then

$$
\begin{gathered}
F=\frac{\cot (\xi, \alpha)}{1 \pm \csc (\xi, \alpha)}, \quad F=\sec (\xi, \alpha)-\tan (\xi, \alpha), \\
F=\cot (\xi, \alpha) \pm \csc (\xi, \alpha) .
\end{gathered}
$$

Case 7. If $A=1$ and $B=-4$, then

$$
F=\frac{\tanh (\xi, \alpha)}{1+\tanh ^{2}(\xi, \alpha)} .
$$

Case 8. If $A=1$ and $B=4$, then

$$
F=\frac{\tan (\xi, \alpha)}{1-\tan ^{2}(\xi, \alpha)}
$$

Case 9. If $A=-1$ and $B=-4$, then

$$
F=\frac{\cot (\xi, \alpha)}{1-\cot ^{2}(\xi, \alpha)}
$$

where the generalized hyperbolic and trigonometric functions are defined as

$$
\begin{gathered}
\cosh (\xi, \alpha)=\frac{E_{\alpha}\left(\xi^{\alpha}\right)+E_{\alpha}\left(-\xi^{\alpha}\right)}{2}, \\
\sinh (\xi, \alpha)=\frac{E_{\alpha}\left(\xi^{\alpha}\right)-E_{\alpha}\left(-\xi^{\alpha}\right)}{2}, \\
\cos (\xi, \alpha)=\frac{E_{\alpha}\left(i \xi^{\alpha}\right)+E_{\alpha}\left(-i \xi^{\alpha}\right)}{2}, \\
\sin (\xi, \alpha)=\frac{E_{\alpha}\left(i \xi^{\alpha}\right)-E_{\alpha}\left(-i \xi^{\alpha}\right)}{2 i}, \\
\tanh (\xi, \alpha)=\frac{\sinh (\xi, \alpha)}{\cosh (\xi, \alpha)}, \quad \tan (\xi, \alpha)=\frac{\sin (\xi, \alpha)}{\cos (\xi, \alpha)} . \\
\operatorname{coth}(\xi, \alpha)=\frac{1}{\tanh (\xi, \alpha)}, \quad \operatorname{sech}(\xi, \alpha)=\frac{1}{\cosh (\xi, \alpha)}, \\
\cot (\xi, \alpha)=\frac{1}{\tan (\xi, \alpha)}, \quad \sec (\xi, \alpha)=\frac{1}{\cos (\xi, \alpha)}, \\
\operatorname{csch}(\xi, \alpha)=\frac{1}{\sinh (\xi, \alpha)}, \quad \csc (\xi, \alpha)=\frac{1}{\sin (\xi, \alpha)} .
\end{gathered}
$$

Determining the integer $n$ and substituting (6) with (7) into (5) and collecting all terms with the same order of $F(\xi)$, then setting each coefficient of $F(\xi)$ to zero yields a system of overdetermined nonlinear fractional equations for $f(t), a_{i}(t)$, and $k$. Solving this system for $f(t), a_{i}(t)$, and $k$, we get explicit expressions for $a_{i}(t)$ and $\xi$. Using the solutions of (7) into (6), we obtain exact solutions of FDE (5).

Remark 1. It can be easily found that if $a_{i}(t)$ are constants and $\xi$ is linear function of $x$ and $t$, then (6) becomes the same as (6) constructed in [28]. Therefore, we may get more general exact solutions of the FDE (5).

\section{Solution of Space-Time Fractional Burger's Equation}

To solve the space-time fractional Burger's equation (1) with moving boundary (2), firstly, we apply the following transformation to (1):

$$
u(x, t)=U(y, t), \quad x=y+s(t) .
$$

This transformation maps equation (1) into the following forced space-time Burger's equation:

$$
D_{t}^{\alpha} U-\sigma D_{y}^{2 \alpha} U-2 \mu U D_{y}^{\alpha} U=\left(s^{\prime}(t)\right)^{\alpha} D_{y}^{\alpha} U,
$$

with initial-boundary conditions

$$
U(y, 0)=h(y), \quad U(0, t)=g(t),
$$

where $s^{\prime}(t)=d s / d t$ and $s(0)=0$, which implies the compatibility condition $g(0)=u(0,0)$. The moving boundary 
problem (1)-(2) of the fractional space-time Burger's equation is transformed into a fixed boundary problem with forced nonlinear fractional differential equation (16) in the quarter plane $y \geq 0, t \geq 0$ with boundary condition (17). In order to solve (16) by the fractional Riccati expansion method, we use the following transformation:

$$
U(y, t)=U(\xi), \quad \xi=k y+f(t) .
$$

In particular, when $f(t)=\omega t$ then $\xi$ represents a travelling wave with velocity $\omega$. By applying (18) into (16), we obtain the following nonlinear fractional ordinary differential equation:

$$
\left(f^{\prime}\right)^{\alpha} D_{\xi}^{\alpha} U-\sigma k^{2 \alpha} D_{\xi}^{2 \alpha} U-\mu k^{\alpha} U D_{\xi}^{\alpha} U=\left(s^{\prime}(t)\right)^{\alpha} k^{\alpha} D_{\xi}^{\alpha} U,
$$

where $f^{\prime}=d f / d t$. Balancing $D_{\xi}^{2 \alpha} u$ with $u D_{\xi}^{\alpha} u$ gives $n=1$. Therefore, the solution of (16) is expressed as

$$
U=a_{0}(t)+a_{1}(t) F(\xi) .
$$

Substituting (20) into (19) using (7) and setting the coefficients of $F(\xi)$ to zero, we get

$$
\left(f^{\prime}\right)^{\alpha}=k^{\alpha}\left[\left(s^{\prime}\right)^{\alpha}+2 \mu a_{0}\right], \quad a_{1}=-\frac{\sigma k^{\alpha} B}{\mu},
$$

where $a_{0}$ and $k$ are arbitrary constants. Therefore, general formula of the solution of the forced space-time fractional Burger's equation (16) is

$$
\begin{aligned}
U(y, t) & =a_{0}-\frac{\sigma k^{\alpha} B}{\mu} F(k y+f(t)), \\
\left(f^{\prime}\right)^{\alpha} & =k^{\alpha}\left[\left(s^{\prime}\right)^{\alpha}+2 \mu a_{0}\right] .
\end{aligned}
$$

From the solutions of (7), by choosing the special values of $A, B$, and the corresponding function $F(\xi)$, we get the following solutions of (16):

$$
\begin{gathered}
U_{1}(y, t)=a_{0}-\frac{\sigma k^{\alpha}}{\mu} \tan (k y+f(t), \alpha), \\
\left(f^{\prime}\right)^{\alpha}=k^{\alpha}\left[\left(s^{\prime}\right)^{\alpha}+2 \mu a_{0}\right] \\
U_{2}(y, t)=a_{0}+\frac{\sigma k^{\alpha}}{\mu} \tanh (k y+f(t), \alpha), \\
\left(f^{\prime}\right)^{\alpha}=k^{\alpha}\left[\left(s^{\prime}\right)^{\alpha}+2 \mu a_{0}\right] \\
U_{3}(y, t)=a_{0}+\frac{\sigma k^{\alpha}}{2 \mu}\left[\frac{\tanh (k y+f(t), \alpha)}{1 \pm \operatorname{sech}(k y+f(t), \alpha)}\right] \\
\left(f^{\prime}\right)^{\alpha}=k^{\alpha}\left[\left(s^{\prime}\right)^{\alpha}+2 \mu a_{0}\right] .
\end{gathered}
$$

The remaining solutions can be obtained in a similar manner. When $\alpha=1$, we obtain the following forced Burger's equation (see [56-58]):

$$
U_{t}-\sigma U_{y y}-2 \mu U U_{y}=s^{\prime}(t) U_{y}
$$

Thus, the solutions (23) are reduced to the solutions of (24), which are given by

$$
\begin{gathered}
U_{1 \mathrm{fbur}}=a_{0}-\frac{\sigma k}{\mu} \tan \left(k\left(y+s(t)+2 \mu a_{0} t\right)\right), \\
U_{2 \mathrm{fbur}}=a_{0}+\frac{\sigma k}{\mu} \tanh \left(k\left(y+s(t)+2 \mu a_{0} t\right)\right), \\
U_{3 \mathrm{fbur}}=a_{0}+\frac{\sigma k}{2 \mu}\left[\frac{\tanh \left(k\left(y+s(t)+2 \mu a_{0} t\right)\right)}{1 \pm \operatorname{sech}\left(k\left(y+s(t)+2 \mu a_{0} t\right)\right)}\right] .
\end{gathered}
$$

Meanwhile, from solutions (25)-(27), we see that the velocity of the wave is related to the moving boundary function $s(t)$. To the best of our knowledge some of the obtained solutions appear for the first time concerning this problem. In order to understand the evolution of the solutions (25)(27), the main soliton features of them are investigated by using direct computer simulations with the accuracy $10^{-9}$. We discuss the effect of the moving boundary condition in some special cases, such as linear and periodic moving boundary to the kink soliton solution (26).

Firstly, we study the features of the solution (26) for linear moving boundary. In order to do that we choose

$$
s(t)=v t
$$

Taking $\sigma=\mu=-1, a_{0}=k=1$, then, from (17) and (26), the initial-boundary condition is given by

$$
g(t)=1+\tanh ((v-2) t), \quad h(y)=1+\tanh (y) \text {. }
$$

Note that in the static case when $v \rightarrow 0$, solution (26) becomes kink solution with velocity $2 \mu a_{0}$. Figure 1 shows the solution (26) of the forced Burger's equation (24) in the moving frame of reference for a linear moving boundary (28), with $v=100,-100,0.001,-0.001$. The kink behavior appears clearly when the absolute value of $v$ is sufficiently small as shown in Figures 1(c) and 1(d). The velocity of the boundary in the moving frame moves in a concave shape as shown in Figure 1(a), whilst moves in a convex shape as shown in Figure 1(b) in the $t$-direction. Also, the velocity of the boundary in the moving frame is shown as a convex shape in the $t$-direction in Figures 1(c) and 1(d).

In the special case of a linear moving boundary, the solution of the Burger's equation can be found directly from (24) via a change of dependent variable. In fact, if $s(t)=v t$, then (24) becomes

$$
U_{t}-\sigma U_{y y}-2 \mu U U_{y}=v U_{y}
$$

By taking the dependent variable to be

$$
w(y, t)=U(y, t)+\frac{v}{2 \mu}
$$

then (30) becomes the Burger's equation

$$
w_{t}=\sigma w_{y y}+2 \mu w w_{y}
$$




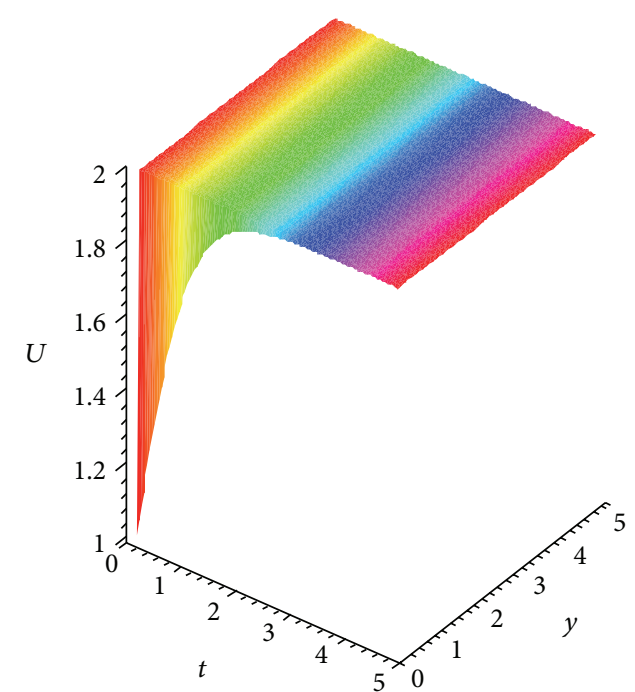

(a)

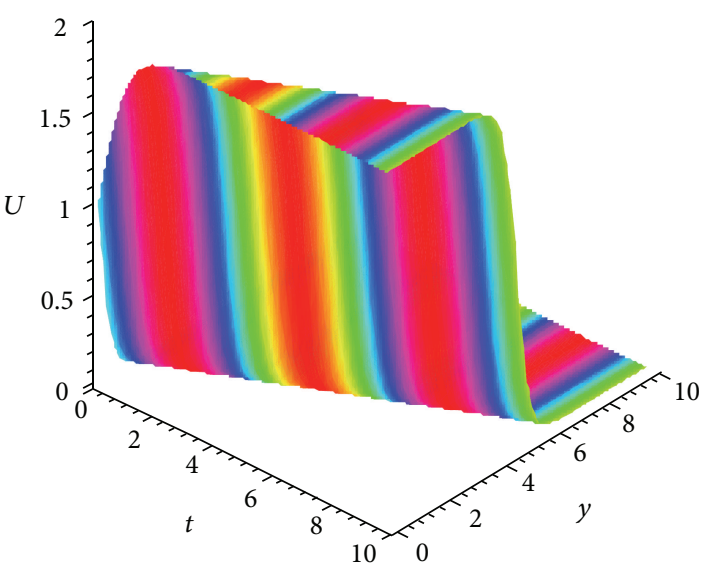

(c)

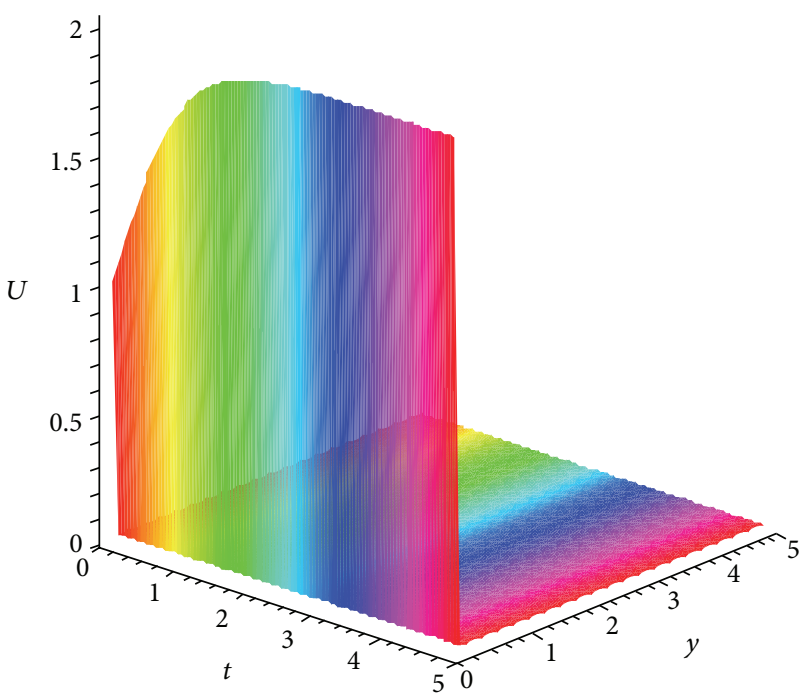

(b)

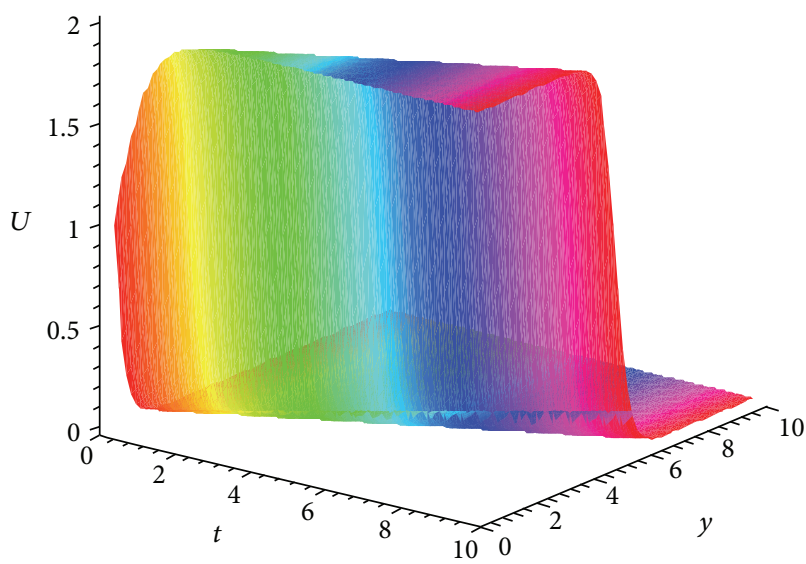

(d)

FIGURE 1: Evolutional behavior of $U_{2}$ describes kink soliton solution with linear moving boundary $s(t)=v t$ and $\sigma=\mu=-1, a_{0}=k=1:(\mathrm{a})$ $v=100$; (b) $v=-100$; (c) $v=0.001$; (d) $v=-0.001$.

with the initial-boundary conditions

$$
w(y, 0)=h(y)+\frac{v}{2 \mu}, \quad w(0, t)=g(t)+\frac{v}{2 \mu}
$$

Secondly, we discuss the features of the kink soliton solution (26) for the periodic moving boundary. Now let us choose

$$
s(t)=10 \sin (v t) .
$$

The initial-boundary conditions have the form

$$
\begin{aligned}
& g(t)=1+\tanh (10 \sin (v t)-2 t), \\
& h(y)=1+\tanh (y) .
\end{aligned}
$$

Figure 2 presents the surface plot of solution (26) for the forced Burger's equation (24) with periodic moving boundary (34) for $v=1$ and $v=10$. Because of the periodic property of the moving boundary $s(t)$, the kink-type soliton propagation of the solution (26) shows the periodic property.

When $s(t)=10 \sin (v t),(24)$ becomes

$$
U_{t}-\sigma U_{y y}-2 \mu U U_{y}=10 v \cos (v t) U_{y}
$$

Considering the transformation

$$
w(y, t)=U(y, t)+\frac{5 v}{\mu} \cos (v t)
$$

then (36) is converted into the Burger's equation

$$
w_{t}=\sigma w_{y y}+2 \mu w w_{y}
$$




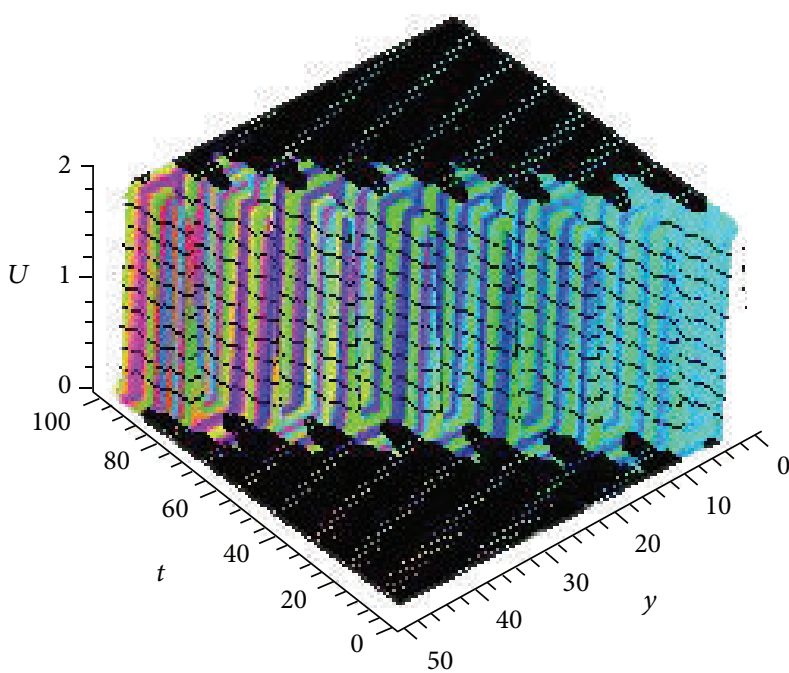

(a)

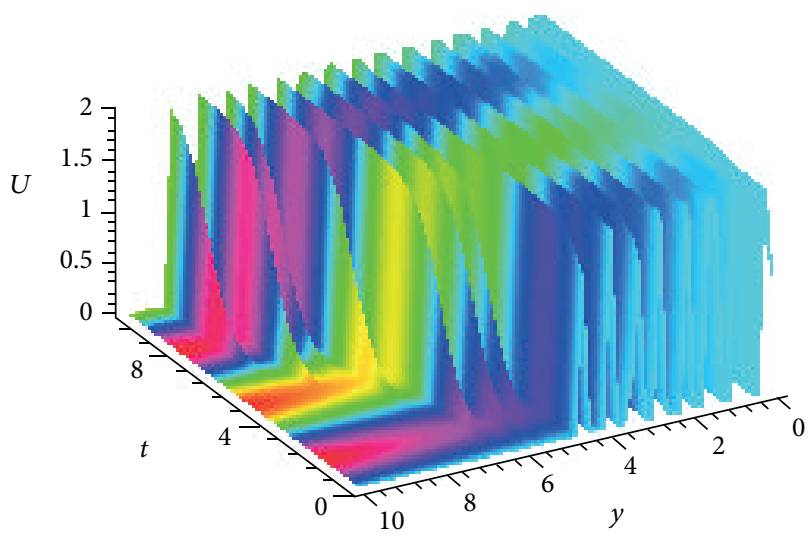

(c)

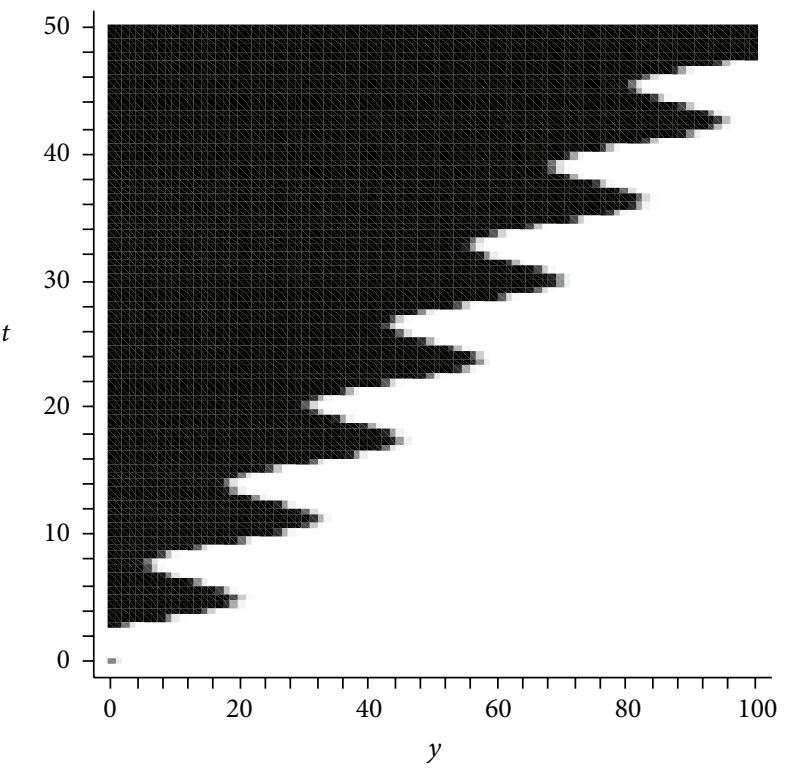

(b)

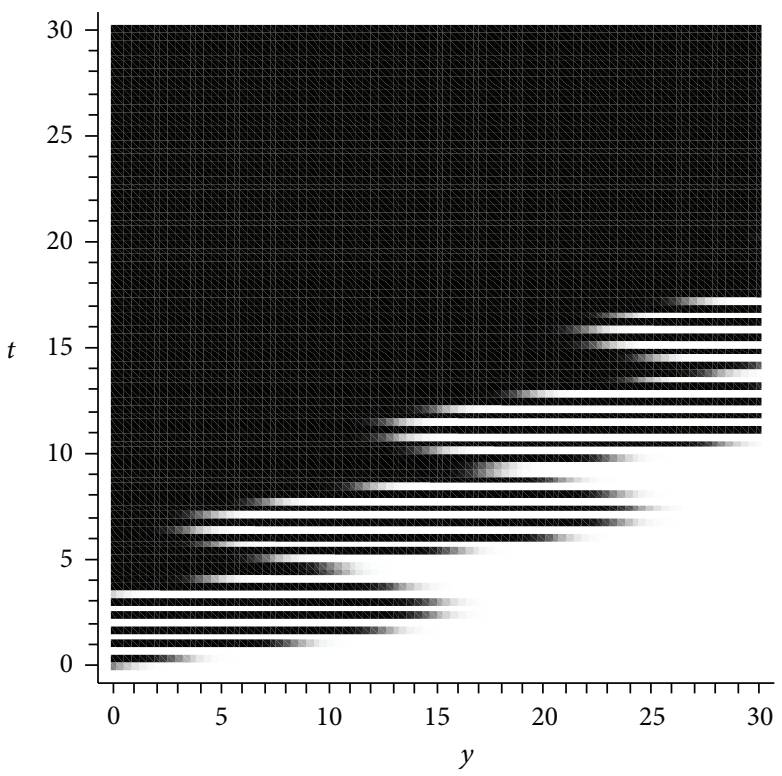

(d)

FIGURE 2: Evolutional behavior of $U_{2}$ describes kink soliton solution with periodic moving boundary $s(t)=10 \sin (v t)$ : (a) $v=1$; (b) density plot of (a); (c) $v=10$; (d) density plot of (c).

with the initial-boundary conditions

$$
w(y, 0)=h(y)+\frac{5 v}{\mu}, \quad w(0, t)=g(t)+\frac{5 v}{\mu} \cos (v t) .
$$

\section{Conclusions}

In this paper, the fractional Riccati expansion method with variable coefficients has been successfully applied to the forced space-time fractional Burger's equation. A number of new analytical solutions have been obtained. Figures 1 and 2 show the solutions of the forced Burger's equation with linear and periodic moving boundaries at $\alpha=1$. The variable coefficient fractional Riccati expansion method can be applied to other nonlinear FDEs with variable coefficients.

\section{Conflict of Interests}

The authors declare that there is no conflict of interests regarding the publication of this paper. 


\section{References}

[1] A. A. Kilbas, H. M. Srivastava, and J. J. Trujillo, Theory and Applications of Fractional Differential Equations, Elsevier, Elsevier, Amsterdam, The Netherlands, 2006.

[2] B. J. West, M. Bolognab, and P. Grigolini, Physics of Fractal Operators, Springer, New York, NY, USA, 2003.

[3] S. G. Samko, A. A. Kilbas, and O. I. Marichev, Fractional Integrals and Derivatives: Theory and Applications, Gordon and Breach, Yverdon, Switzerland, 1993.

[4] J. H. He, "Some applications of nonlinear fractional differential equations and their approximations," Bulletin of Science, Technology \& Society, vol. 15, no. 2, pp. 86-90, 1999.

[5] M. Cui, "Compact finite difference method for the fractional diffusion equation," Journal of Computational Physics, vol. 228, no. 20, pp. 7792-7804, 2009.

[6] A. M. A. El-Sayed and M. Gaber, "The Adomian decomposition method for solving partial differential equations of fractal order in finite domains," Physics Letters A, vol. 359, no. 3, pp. 175-182, 2006.

[7] A. M. A. El-Sayed, S. H. Behiry, and W. E. Raslan, "Adomian's decomposition method for solving an intermediate fractional advection-dispersion equation," Computers \& Mathematics with Applications, vol. 59, no. 5, pp. 1759-1765, 2010.

[8] Z. Odibat and S. Momani, "A generalized differential transform method for linear partial differential equations of fractional order," Applied Mathematics Letters, vol. 21, no. 2, pp. 194-199, 2008.

[9] J. H. He, "A new approach to nonlinear partial differential equations," Communications in Nonlinear Science and Numerical Simulation, vol. 2, no. 4, pp. 230-235, 1997.

[10] D. Baleanu, K. Diethelm, E. Scalas, and J. J. Trujillo, Fractional Calculus: Models and Numerical Methods, vol. 3 of Series on Complexity, Nonlinearity and Chaos, World Scientific, Hackensack, NJ, USA, 2012.

[11] I. Podlubny, Fractional Differential Equations, vol. 198, Academic Press, San Diego, Calif, USA, 1999.

[12] S. G. Samko, A. A. Kilbas, and O. I. Marichev, Fractional Integrals and Derivatives: Theory and Applications, Gordon and Breach Science Publishers, Amsterdam, The Netherlands, 1993.

[13] G. Jumarie, "On the fractional solution of the equation $f(x+y)$ $=f(x) f(y)$ and its application to fractional Laplace's transform," Applied Mathematics and Computation, vol. 219, no. 4, pp. 16251643, 2012.

[14] G. Jumarie, "An approach via fractional analysis to non-linearity induced by coarse-graining in space," Nonlinear Analysis. Real World Applications, vol. 11, no. 1, pp. 535-546, 2010.

[15] G. Jumarie, "Table of some basic fractional calculus formulae derived from a modified Riemann-Liouville derivative for nondifferentiable functions," Applied Mathematics Letters, vol. 22, no. 3, pp. 378-385, 2009.

[16] G.-W. Wang, X.-Q. Liu, and Y.-Y. Zhang, "Lie symmetry analysis to the time fractional generalized fifth-order KdV equation," Communications in Nonlinear Science and Numerical Simulation, vol. 18, no. 9, pp. 2321-2326, 2013.

[17] H. Wang and T.-C. Xia, "The fractional supertrace identity and its application to the super Jaulent-Miodek hierarchy," Communications in Nonlinear Science and Numerical Simulation, vol. 18, no. 10, pp. 2859-2867, 2013.

[18] Z. Hammouch and T. Mekkaoui, "Travelling-wave solutions for some fractional partial differential equation by means of generalized trigonometry functions," Internationaal Journal of Applied Mathematical Research, vol. 1, no. 2, pp. 206-212, 2012.

[19] R. Almeida and D. F. M. Torres, "Fractional variational calculus for nondifferentiable functions," Computers and Mathematics with Applications, vol. 61, no. 10, pp. 3097-3104, 2011.

[20] G.-C. Wu, "A fractional variational iteration method for solving fractional nonlinear differential equations," Computers and Mathematics with Applications, vol. 61, no. 8, pp. 2186-2190, 2011.

[21] S. Zhang, Q. A. Zong, D. Liu, and Q. Gao, "A generalized expfunction method for fractional Riccati differential equations,", Communications in Fractional Calculus, vol. 1, no. 1, pp. 48-51, 2010.

[22] B. Tang, Y. He, L. Wei, and X. Zhang, "A generalized fractional sub-equation method for fractional differential equations with variable coefficients," Physics Letters A, vol. 376, no. 38-39, pp. 2588-2590, 2012.

[23] B. Zheng, “ $\left(G^{\prime} / G\right)$-expansion method for solving fractional partial differential equations in the theory of mathematical physics," Communications in Theoretical Physics, vol. 58, no. 5, pp. 623-630, 2012.

[24] A. Akgül, A. Kılıçman, and M. Inc, "Improved (G'/G)expansion method for the space and time fractional foam drainage and KdV equations," Abstract and Applied Analysis, vol. 2013, Article ID 414353, 7 pages, 2013.

[25] B. Lu, "The first integral method for some time fractional differential equations," Journal of Mathematical Analysis and Applications, vol. 395, no. 2, pp. 684-693, 2012.

[26] S. Zhang and H. Zhang, "Fractional sub-equation method and its applications to nonlinear fractional PDEs," Physics Letters. A, vol. 375, no. 7, pp. 1069-1073, 2011.

[27] S. Guo, L. Mei, Y. Li, and Y. Sun, "The improved fractional sub-equation method and its applications to the space-time fractional differential equations in fluid mechanics," Physics Letters A, vol. 376, no. 4, pp. 407-411, 2012.

[28] E. A. Abdel-Salam and E. A. Yousif, "Solution of nonlinear space-time fractional differential equations using the fractional Riccati expansion method," Mathematical Problems in Engineering, vol. 2013, Article ID 846283, 6 pages, 2013.

[29] W. Li, H. Yang, and B. He, "Exact solutions of fractional burgers and Cahn-Hilliard equations using extended fractional Riccati expansion method," Mathematical Problems in Engineering, vol. 2014, Article ID 104069, 9 pages, 2014.

[30] A. Friedman, Partial Differential Equations of Parabolic Type, Prentice Hall, Englewood Cliffs, NJ, USA, 1964.

[31] A. Friedman, Variational Principles and Free-Boundary Problems, John Wiley \& Sons, New York, NY, USA, 1982.

[32] M. A. Hastaoglu, "Numerical solution of three-dimensional moving boundary problems: melting and solidification with blanketing of a third layer," Chemical Engineering Science, vol. 42, no. 10, pp. 2417-2423, 1987.

[33] M. Zerroukat and C. R. Chatwin, "Explicit variable time-step method for implicit moving boundary problems," Communications in Numerical Methods in Engineering, vol. 10, no. 3, pp. 227-235, 1994.

[34] P. J. Slikkerveer, E. P. van Lohuizen, and S. B. G. O’Brien, “An implicit surface tension algorithm for picard solvers of surfacetension-dominated free and moving boundary problems," International Journal for Numerical Methods in Fluids, vol. 22, no. 9, pp. 851-865, 1996. 
[35] N. Bodard, R. Bouffanais, and M. O. Deville, "Solution of moving-boundary problems by the spectral element method," Applied Numerical Mathematics, vol. 58, no. 7, pp. 968-984, 2008.

[36] R. Kannan and Z. J. Wang, "A high order spectral volume method for moving boundary problems," in Proceedings of the 40th AIAA Fluid Dynamics Conference, Chicago, Ill, USA, July 2010.

[37] W. Cao, W. Huang, and R. D. Russell, "A moving mesh method based on the geometric conservation law," SIAM Journal on Scientific Computing, vol. 24, no. 1, pp. 118-142, 2002.

[38] R. Li, T. Tang, and P. Zhang, "A moving mesh finite element algorithm for singular problems in two and three space dimensions," Journal of Computational Physics, vol. 177, no. 2, pp. 365393, 2002.

[39] M. J. Baines, M. E. Hubbard, and P. K. Jimack, "A moving mesh finite element algorithm for fluid flow problems with moving boundaries," International Journal for Numerical Methods in Fluids, vol. 47, no. 10-11, pp. 1077-1083, 2005.

[40] M. J. Baines, M. E. Hubbard, P. K. Jimack, and A. C. Jones, "Scale-invariant moving finite elements for nonlinear partial differential equations in two dimensions," Applied Numerical Mathematics., vol. 56, no. 2, pp. 230-252, 2006.

[41] G. A. Markou, Z. S. Mouroutis, D. C. Charmpis, and M. Papadrakakis, "The ortho-semi-torsional (OST) spring analogy method for 3D mesh moving boundary problems," Computer Methods in Applied Mechanics and Engineering, vol. 196, no. 46, pp. 747-765, 2007.

[42] M. E. Hubbard, M. J. Baines, and P. K. Jimack, "Consistent Dirichlet boundary conditions for numerical solution of moving boundary problems," Applied Numerical Mathematics, vol. 59, no. 6, pp. 1337-1353, 2009.

[43] S. G. Ahmed and S. A. Meshrif, "A new numerical algorithm for 2D moving boundary problems using a boundary element method," Computers \& Mathematics with Applications, vol. 58, no. 7, pp. 1302-1308, 2009.

[44] J. H. Kol, S. H. Park, H. C. Park, and D. Byun, "Finite macro-element-based volume grid deformation for large moving boundary problems," International Journal for Numerical Methods in Biomedical Engineering, vol. 26, no. 12, pp. 16561673, 2010.

[45] W. Liu, J. Yao, and Y. Wang, "Exact analytical solutions of moving boundary problems of one-dimensional flow in semiinfinite long porous media with threshold pressure gradient," International Journal of Heat and Mass Transfer, vol. 55, no. 2122, pp. 6017-6022, 2012.

[46] C. Atkinson, "Moving boundary problems for time fractional and composition dependent diffusion," Fractional Calculus \& Applied Analysis, vol. 15, no. 2, pp. 207-221, 2012.

[47] C. Yin and M. Xu, "An asymptotic analytical solution to the problem of two moving boundaries with fractional diffusion in one-dimensional drug release devices," Journal of Physics A: Mathematical and Theoretical, vol. 42, no. 11, Article ID 115210, 2009.

[48] M. S. Kushwaha and A. Kumar, "An approximate solution to a moving boundary problem with space-time fractional derivative in fluvio-deltaic sedimentation process," Ain Shams Engineering Journal, vol. 4, pp. 889-895, 2013.

[49] X. Li, S. Wang, and M. Zhao, "Two methods to solve a fractional single phase moving boundary problem," Central European Journal of Physics, vol. 11, no. 10, pp. 1387-1391, 2013.
[50] H. Brezis and F. Browder, "Partial differential equations in the 20th century," Advances in Mathematics, vol. 135, no. 1, pp. 76$144,1998$.

[51] A. D. Polyanin and V. F. Zaitsev, Handbook of Nonlinear Partial Differential Equations, Chapman \& Hall/CRC, Boca Raton, Fla, USA, 2004.

[52] W. Malfliet, "Solitary wave solutions of nonlinear wave equations," American Journal of Physics, vol. 60, no. 7, pp. 650-654, 1992.

[53] P. G. Estévez and P. R. Gordoa, "Non classical symmetries and the singular manifold method. Theory and six examples," Studies in Applied Mathematics, vol. 95, pp. 73-113, 1995.

[54] S. D. Liu, S. K. Liu, and Q. X. Ye, "Explicit traveling wave solutions of nonlinear evolution equations," Mathematics in Practice and Theory, vol. 28, no. 4, pp. 289-301, 1998.

[55] A. M. Wazwaz, "Travelling wave solutions of generalized forms of BURgers, BURgers-KdV and BURgers-Huxley equations," Applied Mathematics and Computation, vol. 169, no. 1, pp. 639656, 2005.

[56] S. D. Lillo, "Moving boundary problems for the Burgers equation," Inverse Problems, vol. 14, pp. L1-L4, 1998.

[57] G. Biondini and S. de Lillo, "On the Burgers equation with moving boundary," Physics Letters A, vol. 279, no. 3-4, pp. 194206, 2001.

[58] F. Calogero and S. D. Lillo, "The Burgers equation on the semiinfinite and finite intervals," Nonlinearty, vol. 2, pp. 37-43, 1987.

[59] G. Jumarie, "Modified Riemann-Liouville derivative and fractional Taylor series of nondifferentiable functions further results," Computers \& Mathematics with Applications, vol. 51, no. 9-10, pp. 1367-1376, 2006.

[60] G. Jumarie, "New stochastic fractional models for Malthusian growth, the Poissonian birth process and optimal management of populations," Mathematical and Computer Modelling, vol. 44, no. 3-4, pp. 231-254, 2006.

[61] G. Jumarie, "Laplace's transform of fractional order via the Mittag-Leffler function and modified Riemann-Liouville derivative," Applied Mathematics Letters, vol. 22, no. 11, pp. 1659$1664,2009$. 


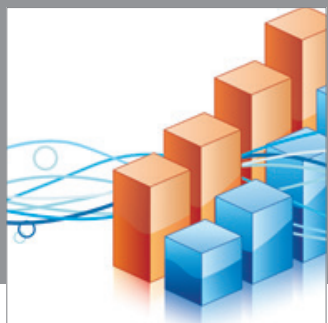

Advances in

Operations Research

mansans

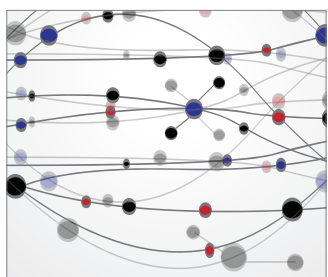

The Scientific World Journal
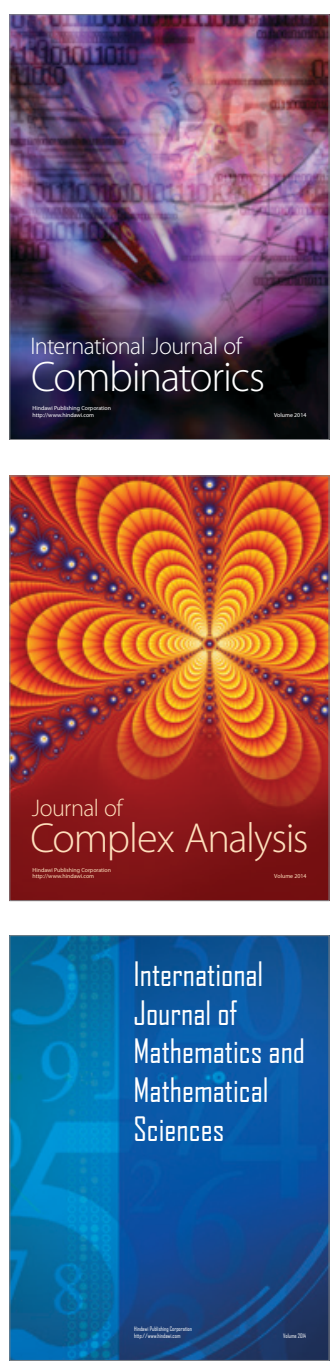
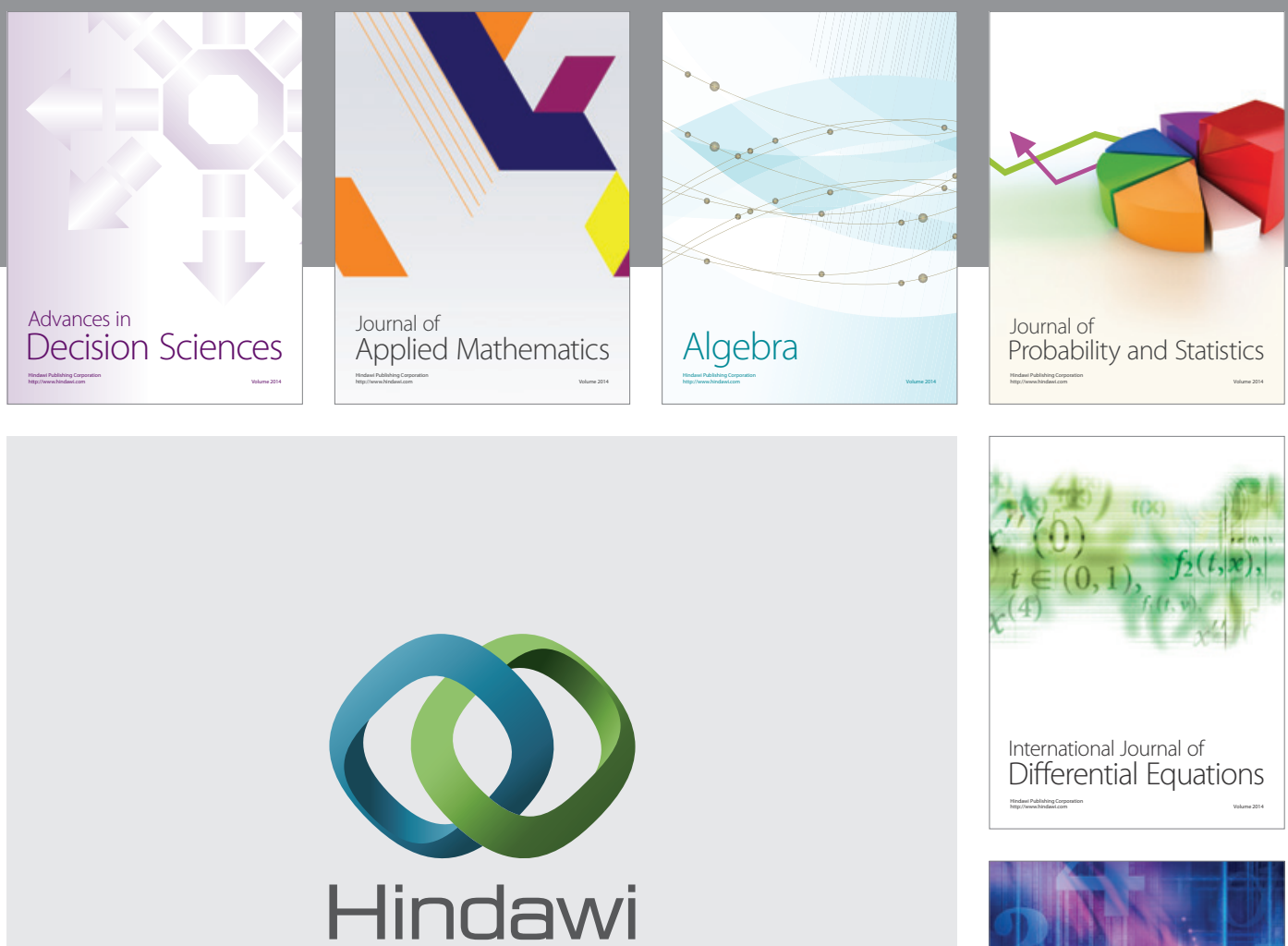

Submit your manuscripts at http://www.hindawi.com
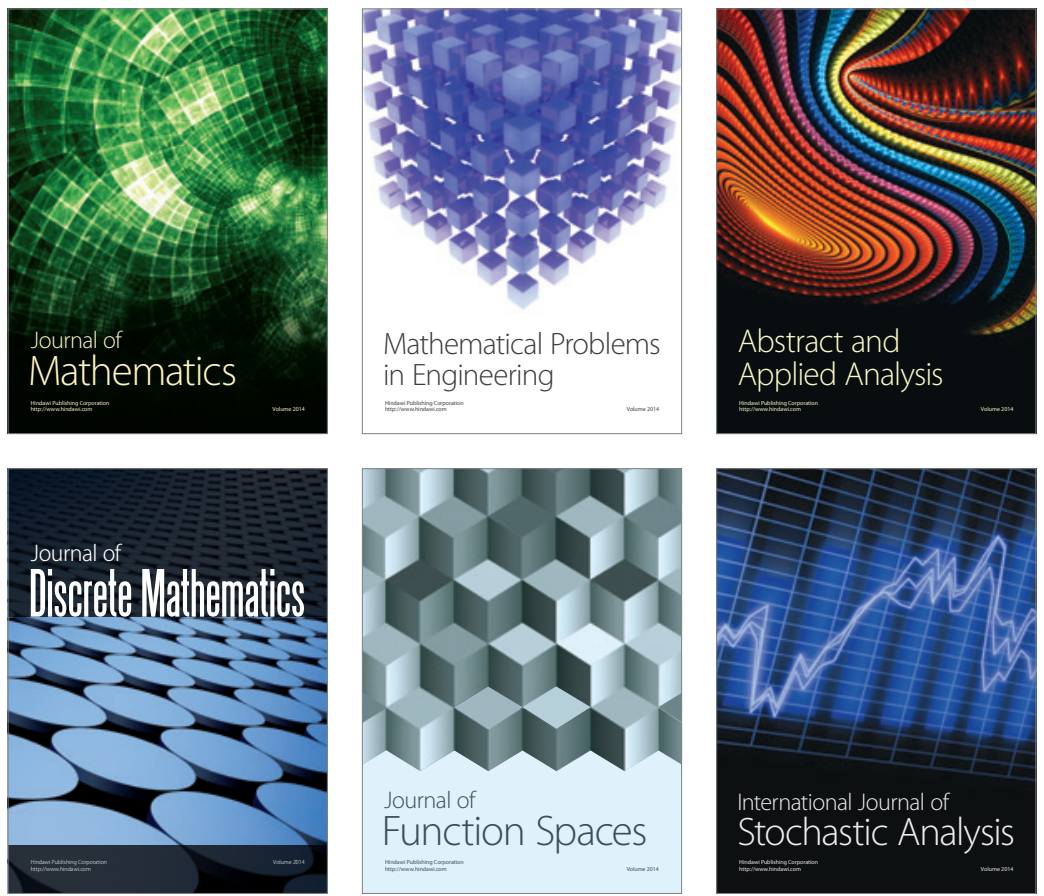

Journal of

Function Spaces

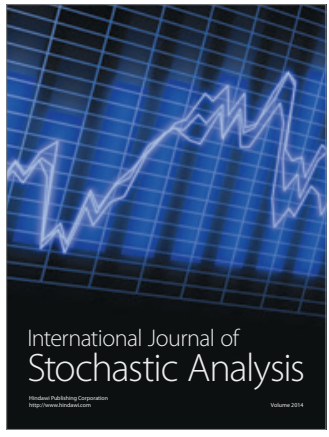

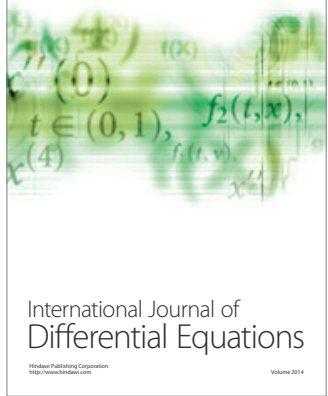
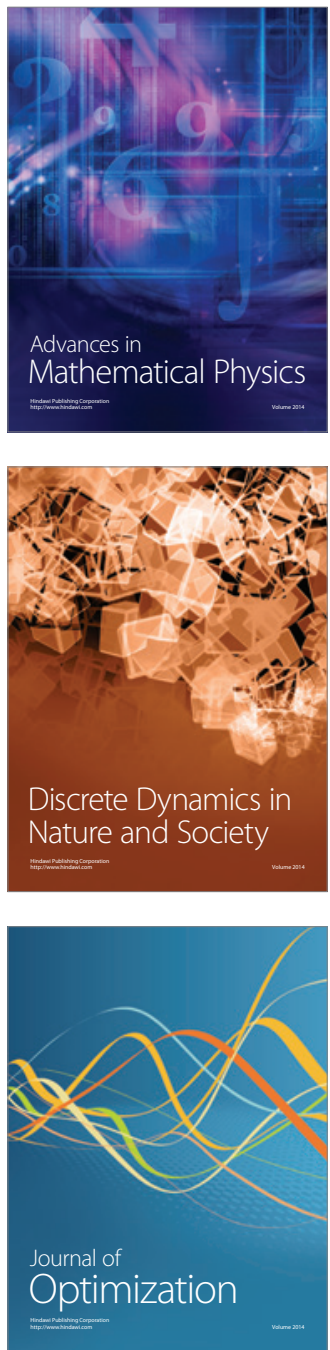